\title{
Aktuelle Fragen zu Diagnostik und Therapie chronisch entzündlicher Darmerkrankungen
}

\author{
Current Issues in Diagnostics and Therapy of Chronic Inflammatory Bowel Diseases \\ Gesprächsleiter: E. Klar, Heidelberg \\ Teilnehmer: A. von Herbay, Heidelberg H.-J. Brambs, Ulm U.R. Fölsch, Kiel \\ P. Layer, Hamburg H.-P. Bruch, H.-B. Gehl, C. Eckmann, Lübeck
}

Frage 1: Ist die Dünndarm-Doppelkontrastuntersuchung nach Sellink im Vergleich zum Hydro-MRT in der Diagnostik des Morbus Crohn noch indiziert?

Brambs: Das Enteroklysma (Dünndarmdoppelkontrast nach Sellink) scheint für die Diagnostik entzündlicher Darmerkrankungen zunehmend an Bedeutung zu verlieren. Nachteile dieser Methode sind die Strahlenexposition und die Notwendigkeit, eine Sonde bis an die Flexura duodenojejunalis platzieren zu müssen. $\mathrm{Ob}$ eine sondenfreie Untersuchung beim MR-Enteroklysma ausreichende Resultate erbringt, ist durch bisherige Studien noch nicht eindeutig belegt. Deutliche Vorteile bietet das MR-Enteroklysma, wenn es um Komplikationen eines Morbus Crohn wie Fisteln, Abszesse und Stenosen geht. Interenterische Prozesse können in verschiedenen Projektionen topographisch übersichtlich abgebildet und charakterisiert werden. So kann mit Hilfe dieser Methode klar unterschieden werden, ob eine Distanzierung von Dünndarmschlingen durch eine Wandverdickung, einen interenterischen Abszess oder eine fibrös-fettige Proliferation bedingt ist. Für die Diagnostik des frühen Stadiums eines Morbus Crohn ist das MR-Enteroklysma unzureichend. Hier besteht noch eine Indikation für das konventionelle Enteroklysma, insbesondere wenn es endoskopisch nicht gelingt, das terminale Ileum zu inspizieren.

Fölsch: Die Dünndarm-Doppelkontrastuntersuchung nach Sellink wird bei uns noch als Standarduntersuchung durchgeführt. Wir haben die Erfahrung gemacht, dass das MRT mit Vanadium-haltigem Kontrastmittel (Ananassaft) in großen Serien in unserer Klinik vergleichbare Resultate liefert. Trotzdem ist die Patientenakzeptanz sehr unterschiedlich. Nicht jeder Patient ist bereit, das MRT der klassischen Untersuchungsmethode nach Sellink vorzuziehen. Gleichzeitig sollte auch herausgestellt werden, dass die Untersuchung nach Sellink in einzelnen Fällen eine bessere Beurteilung des Schleimhautreliefs erlaubt, wohingegen im MRT auch eine Beurteilung des Mesenteriums möglich ist. Eine andere Frage, die durchaus diskutabel wäre, ist, ob die Dünndarmkontrastuntersuchung nach Sellink gegenüber der klassischen MagenDarm-Passage wirklich relevante Vorteile erbringt. Sie ist sehr aufwändig und die Magen-Darm-Passage bringt in Studien ähnlich gute Resultate.

Layer: Das (Hydro-)MRT kann einen Beitrag zur Diagnostik des Morbus Crohn liefern, da sich mit dieser Methodik nicht nur Schleimhautläsionen, sondern auch extraluminale Veränderungen ohne Strahlenbelastung nachweisen lassen können. Aktuelle Studien bewerten die Aussagekraft des (Hydro-) MRT für die Dünndarmdiagnostik des Morbus Crohn als mindestens gleichwertig, überwiegend aber besser im Vergleich zum Enteroklysma. Ich vermute, diese neue Methode wird sich daher mittelfristig durchsetzen und das Enteroklysma ablösen, insbesondere bei schweren Verläufen, um auch relevante extraenterische Komplikationen aufzudecken. Derzeit ist die Methodik beim Morbus Crohn aber noch zu neu, um ihre Wertigkeit außerhalb von Studien abschließend beurteilen zu können. Außerdem ist sie nicht flächendeckend verfügbar.

Bruch/Gehl/Eckmann: Die Röntgenuntersuchung des Dünndarms nach Sellink ist heutzutage nicht mehr als primäre Modalität in der Diagnostik des Morbus Crohn indiziert. Entscheidend für die Frage, ob das konventionelle Enteroklysma ersetzt werden kann, ist die Fähigkeit der MRT, neben extraluminalen Prozessen auch Faltenrelief und Stenosen ähnlich gut darstellen zu können wie die Durchleuchtungsuntersuchung, denn hierbei hat die konventionelle Methode nach Sel-

\begin{tabular}{ll}
\hline KARGER & ๑ 2002 S. Karger GmbH, Freiburg \\
Fax +497614520714 & Accessible online at: \\
$\begin{array}{l}\text { E-mail Information@Karger.de } \\
\text { www.karger.com }\end{array}$ & www.karger.com/journals/cga
\end{tabular}

Prof. Dr. E. Klar

Abteilung für Allgemein-, Viszeral- und Unfallchirurgie Chirurgische Universitätsklinik

Im Neuenheimer Feld 110, D-69120 Heidelberg (Deutschland)

Tel. +49 6221 56-62 04, Fax -57 81

E-mail Ernst_Klar@med.uni-heidelberg.de 
link aufgrund ihrer höheren Auflösung, der dynamischen Beurteilbarkeit und der Möglichkeit zur Palpation zunächst Vorteile. Eine gute Distension des Darms mit intestinalem Kontrastmittel (Wasser, Methylzellulose) wird als wichtigste Voraussetzung für eine ausreichende Beurteilung des Dünndarms in der MRT immer wieder aufgeführt, weil kollabierte Darmschlingen auch große Läsionen markieren und Wandveränderungen vortäuschen können. Wird jedoch die Kontrastierung des Dünndarms über eine Sonde wie beim konventionellen Sellink und gegebenenfalls fraktioniert $(3 \times 400-500$ $\mathrm{ml}$ Methylzellulose) durchgeführt, ist eine optimale und kontrollierte Distension des Dünndarms erreichbar. Die Anatomie des Dünndarms lässt sich in einer der konventionellen Röntgendiagnostik vergleichbaren Qualität darstellen. Darüber hinaus werden Veränderungen der Darmwand und extraluminale Komplikationen wie Abszess- oder Fistelbildungen erkannt. Die Sensitivität der Untersuchung in Bezug auf obstruktive Dünndarmläsionen, aber auch auf geringgradige Läsionen, übertrifft wegen der überlagerungsfreien Darstellung das konventionelle Enteroklysma. Grundsätzlich erscheint die MRT daher als geeignet, das konventionelle Enteroklysma zu ersetzen. Dies gilt insbesondere dann, wenn wiederholte strahlenbelastende Untersuchungen bei jungen Patienten vermieden werden sollen und extraintestinale Komplikationen wie beim Morbus Crohn angenommen werden. Das konventionelle Enteroklysma behält jedoch dort seine Berechtigung, wo kein MRT verfügbar ist. Darüber hinaus ist die konventionelle Darstellung mit geringeren Kosten verbunden.

\section{Frage 2: Welche radiologischen Verfahren erleichtern Ihnen die Abwägung zwischen medikamentöser und chirurgischer Therapie des Morbus Crohn?}

Brambs: Die Frage, ob eine lokale entzündliche Aktivität vorliegt, kann nach intravenöser Gabe von Kontrastmittel an seiner Anreicherung in der verdickten Darmwand abgeschätzt werden. Möglicherweise können dadurch narbige Strikturen, die einer Operation bedürfen, von aktiven entzündlichen Darmwandstenosen differenziert werden, die einer medikamentösen Therapie zugänglich sind. Alternativ könnte für diese Fragestellung auch die farbkodierte Dopplersonographie eingesetzt werden, wenn die fraglichen Darmabschnitte sonographisch gut einzusehen sind.

Fölsch: Die wesentliche Indikation für den Einsatz einer chirurgischen Therapie ist entweder ein therapierefraktärer Status oder eine Komplikation, die nicht mit einer Entzündung zusammenhängt. Therapierefraktärität wird am besten klinisch beurteilt. Die grundsätzliche Detektion von Stenosen ist eine Domäne radiologischer Verfahren. Hier haben wir jedoch auch sehr gute Erfahrungen mit dem Ultraschall gemacht: In der Power-Duplexsonographie ist oft sogar eine
Einschätzung des Entzündungsgrads durch die Darstellung kleiner Gefäße möglich. Nochmals herausstellen möchten wir jedoch, dass die Entscheidung für eine chirurgische Therapie in der Regel auf klinischen Parametern und nicht auf bildgebenden Verfahren oder endoskopischen Befunden beruht.

Layer: Radiologische Verfahren dienen zum Nachweis (oder Ausschluss) spezifischer, oft lokaler Komplikationen chronisch entzündlicher Darmerkrankungen, die chirurgisch behandelt werden (können) (z.B. Fisteln, Perforation, Ileus, intraabdominale Abszedierung, toxisches Megakolon). Je nach Fragestellung kommen hierbei Abdomenübersicht, Kontrastpassagen sowie CT oder MRT in Frage.

Bruch/Gehl/Eckmann: Grundsätzlich ist die MR-Sellink-Technik die Methode der Wahl auch zur Indikation für ein chirurgisches oder konservatives Vorgehen. Lediglich beim so genannten «akuten Abdomen» ist eine Röntgenuntersuchung des Abdomens in 2 Projektionsebenen indiziert, um eine Operationsindikation abzuleiten. In der klinischen Situation eines akuten Ileus ist die hohe Volumenbelastung des Darms durch ein Enteroklysma kontraindiziert. Hier empfiehlt sich die Durchführung einer CT mit intravenöser Kontrastmittelapplikation und nur einer oralen (geringvolumigen) Applikation von jodhaltigem Kontrastmittel.

\section{Frage 3: Wie beurteilen Sie die Kapselendoskopie in der Diagnostik des enteralen Morbus Crohn?}

von Herbay: Die Kapselendoskopie ermöglicht den Einblick in bislang unzugängliche Abschnitte des Dünndarms. Die Wertigkeit jener Befunde im Dünndarm, die mittels Kapselendoskopie jetzt erstmals sichtbar werden, ist derzeit aber noch unklar. Dies gilt vor allem für die Erstdiagnostik eines Morbus Crohn mit isolierter Manifestation im Dünndarm, aber auch für Staging- und Verlaufsuntersuchungen von Patienten mit bereits etablierter Morbus-Crohn-Diagnose. Die Ergebnisse von entsprechenden Studien sollten abgewartet werden.

Brambs: Die Kapselendoskopie wird man nicht einsetzen, wenn entzündliche Strikturen zu befürchten sind. Die Hauptindikation wird die Suche nach okkulten Blutungsquellen sein.

Fölsch: Die Rolle der Kapselendoskopie in der Diagnostik des enteralen Morbus Crohn ist vollständig unklar. Nach eigener Erfahrung ist in jedem Fall der Ausschluss auch entzündlicher Stenosen durch andere Verfahren (Sellink/MRT) zuvor notwendig. Selbst bei mäßiggradigen Stenosierungen kann es zu ganz erheblichen Passageproblemen mit der Kapsel kommen. Sollten sich in der Kapselendoskopie, wie bei uns oft beob- 
achtet, dann verschiedene kleinere Läsionen auch in den proximalen Anteilen des Dünndarms darstellen, so ist deren klinische Signifikanz nicht klar. Damit stellt die weitere Exploration der Kapselendoskopie zwar ein wissenschaftlich interessantes Feld dar, ist jedoch zum jetzigen Zeitpunkt noch nicht im Stadium des diagnostisch relevanten Einsatzes. Nach wie vor gilt: Die Therapie des Morbus Crohn muss sich an der Klinik orientieren! Die diagnostische Rolle der Kapselendoskopie sehen wir im Bereich des Dünndarms im Wesentlichen in der Lokalisation von Blutungsquellen.

Layer: Die Kapselendoskopie kann im Einzelfall bei dringendem Verdacht auf einen enteralen Morbus Crohn und unauffälliger sonstiger bildgebender Diagnostik auch diskrete, radiologisch unsichtbare Schleimhautläsionen aufdecken und so diagnostisch wegweisend sein. Allerdings ist unbedingt zu beachten, dass enterale Stenosen eine Kontraindikation für diese Untersuchungstechnik darstellen und vor Einsatz der Kapselendoskopie mittels Enteroklysma (oder ähnlichem) ausgeschlossen werden müssen. Auf die fehlende Möglichkeit zur Biopsie muss ebenfalls hingewiesen werden. Trotz vielversprechender Kasuistiken und Abstracts liegen bisher nur wenige abgeschlossene, prospektiv kontrollierte Studien als ausführliche Publikationen vor, so dass die klinische Wertigkeit der Kapselendoskopie derzeit noch nicht abschließend beurteilt werden kann.

Bruch/Gehl/Eckmann: Ohne Zweifel steht mit der Kapselendoskopie seit kurzem ein Diagnostikum zur Verfügung, welches die Lücke in der nichtinvasiven intestinalen Diagnostik zwischen der Gastroskopie und der Koloskopie möglicherweise schließen könnte. Alle bisher verfügbaren Publikationen beschäftigen sich mit dem Wert der Kapselendoskopie in der Diagnostik unklarer Blutungen im Dünndarm. Dort konnte in etwa $50 \%$ der Fälle die Blutungsquelle gefunden werden; ferner blieb die als Referenzmethode durchgeführte Enteroskopie ohne zusätzliche Befunde. Aktuell werden Studien zur Validierung der Methode bei chronisch entzündlichen Darmerkrankungen durchgeführt. Die Kapselendoskopie könnte hier beim Morbus Crohn in der Diagnostik von Stenosen und Blutungen hilfreich sein, die im Jejunum oder oberen Ileum gelegen sind. Wegen der Größe der Kapsel besteht jedoch die Gefahr der Einklemmung im stenosierten Bereich mit konsekutivem Ileus. Dennoch kann ein Gesamteindruck über befallene und nicht befallene Dünndarmabschnitte gewonnen werden. Kleinere Läsionen und Fistelgänge werden jedoch leicht übersehen. Ferner ist eine genaue Lokalisation der pathologischen Befunde zur Zeit nicht möglich. Da die Betriebsdauer zwischen 7 und $8 \mathrm{~h}$ liegt, ist die Aussagekraft im unteren Ileum nicht selten limitiert. Auch bei wohlwollender Beurteilung der Methode wird die Kapselendoskopie die in Frage 1 genannten Standardverfahren nicht ersetzen können. Im Gegenteil, die Indikation beim Morbus Crohn muss mit größter Zurückhaltung gestellt werden.

\section{Frage 4: Wie definieren Sie den Stellenwert von Infliximab in der Behandlung des schweren analen Fistelleidens beim Morbus Crohn?}

Fölsch: Infliximab ist die einzige internistische Therapie mit einem in einer kontrollierten Studie nachgewiesenen Effekt auf das perianale Fistelleiden beim Morbus Crohn. Die Wirkung von Infliximab kann zudem durch eine wiederholte Therapie (alle 8 Wochen) stabilisiert werden. Dies hat die Placebo-kontrollierte, einjährige ACCENT-II-Studie gezeigt. Vor Behandlung mit Infliximab müssen jedoch infektionsbedingte Komplikationen sowohl im systemischen Bereich (Tbc) als auch lokal (Abszesse) ausgeschlossen werden. Dazu sollten ein Röntgen-Thorax, gegebenenfalls ein MerieuxMultitest und ein bildgebendes Verfahren im Bereich des Anorektums (z.B. rektale Endosonographie) durchgeführt werden. Wir haben in der Dauertherapie mit Infliximab sehr erfreuliche und für den Verlauf der Erkrankung ausgesprochen positive Veränderungen gesehen. Gleichzeitig sind uns eine Reihe von Patienten bekannt, bei denen wir mit unseren chirurgischen Partnern einen geeigneten Operationszeitpunkt nach Vortherapie mit Infliximab finden konnten und den Patienten dadurch ein sehr gutes Langzeitergebnis ermöglichten.

Layer: Infliximab kann in definierten Situationen bei einem schweren, sonst therapierefraktären analen Fistelleiden eingesetzt werden; es ist aber keine Routinemethode. Zurückhaltung ist vor allem auch wegen des erheblichen Nebenwirkungsrisikos (gehäuft auch letal verlaufende Infektionen, insbesondere Tuberkulosefälle) geboten und der nur vorübergehenden Wirksamkeit (im Mittel etwa 8-10 Wochen), d.h., es wird kein Ausheilen der Fisteln, sondern oft nur ein kurzfristiger Verschluss erreicht.

Bruch/Gehl/Eckmann: In den letzten Jahren wurden mittels rekombinanter Technologien verschiedene Oligonukleotide, Zytokine und Antikörper in die Behandlung des Morbus Crohn eingeführt. Infliximab, ein TNF- $\alpha$-Antikörper, ist das einzige Therapeutikum dieser Art, das bei therapierefraktärem und fistelndem Morbus Crohn zugelassen ist. Es kann bei schwerem perianalem Fistelleiden des Morbus Crohn als Second-Line-Therapie aufgefasst werden, wenn Steroide und Immunsuppressiva versagt haben. Häufig wird es in einer Dosierung von 5-10 mg/kg KG als dreimalige Infusion nach 0, 2 und 6 Wochen eingesetzt. Nach 12 Wochen Beobachtungszeit sind fast $50 \%$ der Patienten in Vollremission. Nichtraucher sprechen signifikant besser an. Demgegenüber steht eine hohe Rezidivrate von über 75\% nach 1 Jahr. Die Frage, ob hier erneute Infusionen mit Infliximab im Abstand von 4-6 Wochen indiziert sind, ist noch nicht endgültig beantwortet, jedoch lassen sich bereits während der Symptomfreiheit bei Patienten unter Infliximab-Therapie endosonographisch Fisteln nachweisen, die dann nach einem mehr oder weniger langen Zeit- 
raum auch klinisch signifikant werden. Vermutlich verlängert die Kombination von Infliximab mit Immunsuppressiva das symptomfreie Intervall. Schwerwiegende Nebenwirkungen mit Sepsis sind hier jedoch stets zu beachten.

In mehreren Zentren wird bei chronischen Fistelleiden nach Infliximab-Therapie die Indikation zur mikrochirurgischen Fistelexzision gestellt, sofern die Fisteln sanierbar erscheinen. Wenngleich größere Statistiken noch fehlen, sind die berichteten Ergebnisse sehr vielversprechend.

\section{Frage 5: Auf welchen Parametern, neben der Dysplasie, basiert lhre Patientenberatung hinsichtlich der operativen Behandlung bei Colitis ulcerosa?}

Fölsch: Die operative Behandlung der Colitis ulcerosa ist die Proktokolektomie. Dafür gibt es neben der Dysplasie im Wesentlichen drei internistische Indikationen: 1. nicht behandelbare Blutung, 2. therapierefraktäres Krankheitsgeschehen und 3. Nebenwirkung der zur Kontrolle der Krankheitsaktivität eingesetzten Medikamente. Wir versuchen, in der Beratung unserer Patienten einen Operationszeitpunkt zu finden, der früh genug ist, sodass Patienten noch nicht unter einer Kumulation von langjährigen Nebenwirkungen ihrer Therapie sowie systemischen Nebenwirkungen ihrer Grunderkrankung leiden. Es sollte jedoch klar sein, dass sich extraintestinale Manifestationen auch nach Proktokolektomie nicht unbedingt bessern. Insbesondere bei Hautmanifestationen, Vaskulitiden, Arthralgien und der primär sklerosierenden Cholangitis ist bekannt, dass ein Progress unabhängig von der Grunderkrankung auch nach Proktokolektomie erfolgen kann. Die Patienten mit primär sklerosierender Cholangitis beraten wir aufgrund des deutlich erhöhten kolorektalen Karzinomrisikos besonders intensiv. Hier ist ein langjähriger schwerer und refraktärer Verlauf, auch ohne Dysplasie, bereits eine relative Operationsindikation.

Layer: Die Wahrscheinlichkeit der Entwicklung eines Karzinoms bei Colitis ulcerosa nimmt mit der Dauer und der Ausdehnung der Erkrankung zu. Besteht gleichzeitig eine primär sklerosierende Cholangitis, ist das Risiko zusätzlich erhöht. Auch bei Patienten mit anhaltend hoher Morbidität trotz Ausreizen der medikamentösen Behandlung, schweren Nebenwirkungen der konservativen Therapie oder Medikamentenunverträglichkeiten sollte eine operative Therapie langfristig angedacht werden. Bei Kindern und Jugendlichen sind Wachstumsstörungen als zusätzliche Indikation zur elektiven Operation zu beachten. Hinzu kommen die bekannten akuten Komplikationen der Colitis ulcerosa, bei denen dringlich bzw. notfallmäßig operiert werden muss (toxisches Megakolon, Perforation, vital bedrohliche Blutung, fulminanter Schub ohne Ansprechen auf die konservative Therapie).

Bruch/Gehl/Eckmann: Zu den gesicherten Risikofaktoren für die Entstehung eines Colitis-ulcerosa-Karzinoms zählen der frühe Erkrankungsbeginn, die Erkrankungsdauer über 10 Jahre sowie der ausgedehnte Kolonbefall (bei einer Pankolitis ist das Risiko um den Faktor 15 erhöht). Als weiteren Risikofaktor muss man die primär sklerosierende Cholangitis ansehen, die das Risiko auf das bis zu 55fache ansteigen lassen kann. Auch andere extraintestinale Manifestationen sind zu beachten. Im Wesentlichen können zwei große Indikationsgruppen unterschieden werden:

1. Die krankheitsabhängigen Komplikationen. Hier sind das toxische Megakolon, das auf einen 24-stündigen intensivtherapeutischen Versuch nicht anspricht, die Perforation, die Blutung, die wie auch immer geartete Obstruktion und die Dysplasie zu nennen. 2. Daneben sind die Misserfolge der medikamentösen Therapie zu nennen wie Erkrankungsverläufe, die auf die medikamentöse Therapie nicht oder nur unvollständig ansprechen, die hohe Dosen von Steroiden dauerhaft erfordern und die mit Komplikationen auf die Medikation reagieren wie etwa Osteoporose oder Spontanfraktur nach Kortikoidapplikation. Letztendlich kann die Indikation auch dann gegeben sein, wenn die Patienten-Compliance nicht ausreicht. Als weiche Indikationen, über die intensiv interdisziplinär diskutiert werden muss, sind der Patientenwunsch und der Verlust der Lebensqualität unter Therapie anzusehen. Bei allen Überlegungen ist jedoch stets davon auszugehen, dass die Colitis ulcerosa durch die chirurgische Proktokolektomie heilbar ist.

\section{Frage 6: Wie definieren Sie die Indikation zur Proktokolektomie und Pouch-Anlage bei Colitis ulcerosa im Kindesalter?}

Fölsch: Die Indikation zur Proktokolektomie und Pouch-Anlage bei Colitis ulcerosa im Kindesalter sollte sehr sorgfältig gestellt werden. Prinzipiell ist die Operation auch bei Kindern möglich. Schwerwiegende Gedeihstörungen und eine Krankheitsaktivität, die medikamentös nicht kontrolliert werden kann, sind hier Hauptindikationen. Oft wird zu lange gewartet, sodass irreversible Schäden eingetreten sind. Kinder manifestieren unglücklicherweise häufig mit einer Pankolitis, die per se eine schlechtere Prognose als eine Linksseiten- oder auf das Proktorektum beschränkte Kolitis hat.

Layer: Wie bereits erwähnt, stellen bei Kindern und Jugendlichen Wachstumsstörungen eine zusätzliche Indikation zur elektiven Operation dar. Prinzipiell ist aber die Indikation zur Proktokolektomie bei Kindern und Jugendlichen mit Colitis ulcerosa nur selten gegeben, am häufigsten bei konservativ nicht beherrschbarer Blutung.

Bruch/Gehl/Eckmann: Grundsätzlich gelten zum einen die gleichen Indikationen zur totalen Kolektomie wie im Erwachsenenalter. Darüber hinaus kann jedoch die bestehende Mal- 
nutrition bei Colitis ulcerosa zu schwerwiegenden Verzögerungen des Wachstums und der Sexualentwicklung führen. Diese Störungen, die zum Teil auch medikamentös induziert sind, erweitern das Indikationsspektrum zur totalen Kolektomie im Kindesalter, speziell während der Adoleszenz. Einer hohen Frühmorbidität von über $20 \%$ nach totaler Kolektomie im Kindesalter stehen gute Langzeitergebnisse gegenüber.

\section{Frage 7: Wie definieren Sie die akute und die chronische Pouchitis?}

von Herbay: Eine akute Pouch-Ileitis ist als aktive Entzündung im Ileum-Pouch zu charakterisieren, zu deren leukozytärem Infiltrat obligat auch neutrophile Granulozyten gehören. Diese kurzlebigen Entzündungszellen rufen in der Ileummukosa meist Kryptenabszesse hervor, deren Sekret dann als Fibrin und Eiter auf die Schleimhautoberfläche gelangen kann. Bei Konfluenz solcher Mikroläsionen können kleine oder gröBere Ulzera entstehen. Diese histopathologischen Kriterien werden im Pouchitis Disease Activity Index (PDAI) entsprechend berücksichtigt.

Die chronische Pouch-Ileitis entspricht einer protrahiert verlaufenden, aktiven Pouch-Ileitis. Von dieser Diagnose abzugrenzen sind die histopathologisch sichtbaren Veränderungen der Mukosa im Ileum-Pouch, welche bei den meisten Patienten im Verlauf von mehreren Monaten eintreten. Abweichend vom präoperativen Ileum kommt es zu einer Adaptation der Mukosa auf die neue physiologische Funktion als Reservoir für einen dünnflüssigen Dünndarmstuhl. Es liegt dann ein neuer Normalzustand vor, aber keine wirkliche Entzündung. Leider wird dies auch heute noch oftmals verkannt, unter anderem im Pouchitis Activity Score (PAS).

Fölsch: Akute und chronische Pouchitis lassen sich nur im Verlauf differenzieren. Während wir bei einer akuten Pouchitis einzelne und voneinander wohl abgrenzbare Episoden kennen, ist eine chronische Pouchitis durch eine Daueraktivität gekennzeichnet. In beiden Fällen sollte eine chirurgische Beschwerdeursache, auch Jahre nach der Operation, sorgfältig ausgeschlossen werden. Ein geeignetes Verfahren dazu ist neben der konsiliarischen Untersuchung zusammen mit dem Chirurgen die rektale Endosonographie.

Layer: Für die Diagnose einer akuten Pouchitis sind die klinische Symptomatik mit erhöhter Stuhlfrequenz, Blutung und Fieber, der endoskopische Befund (Rötung, Ödem, Ulzerationen, Blutungen, Fibrinbeläge), die Histologie (Ulzerationen, Kryptenabszesse, Granulozyteninfiltrate) und die Ergebnisse der klinischen Untersuchung entscheidend. Eine chronische Pouchitis liegt vor, wenn trotz Akuttherapie die Entzündung im Pouch über mehr als 3 Monate anhält und sich histologisch eine subtotale oder totale Zottenatrophie zeigt. Zwischen schwerer Zottenatrophie und Dysplasieentwicklung im Pouch besteht ein Zusammenhang, der durch mehrere Studien gesichert ist.

Bruch/Gehl/Eckmann: Eine Pouchitis tritt nach Kolektomie und ileoanalem Pouch bei Colitis ulcerosa in etwa 30\% der Fälle auf. Bei der akuten Pouchitis muss zwischen der primären (idiopathischen) und der sekundären, d.h. durch chirurgische Komplikationen bedingten Pouchitis differenziert werden. $\mathrm{Zu}$ den chirurgischen Ursachen einer Pouchitis gehören eine Pouch-Fistel, ein Abszess oder eine «outlet obstruction». Beide Formen der akuten Pouchitis können in einen chronischen Zustand übergehen, wenn Symptomatik, endoskopisches Bild und histologische Veränderungen persistieren. Die chronische Pouchitis ist somit gekennzeichnet durch eine moderate oder schwere Entzündungsaktivität im Pouch über mehr als 3 Monate. Etwa 5\% aller Patienten mit ileoanalem Pouch entwickeln eine chronische Pouchitis. Die Intensität von Überwachung und Therapie ist dem Vorgehen bei der akuten Entzündung gleichzusetzen.

\section{Frage 8: Welche Risikokonstellationen definieren Sie bei chronischer Pouchitis hinsichtlich der Entwicklung eines Pouch-Karzinoms?}

von Herbay: Hier sind zwei unterschiedliche Risiken zu betrachten. Zum einen gilt es, das Risiko eines Pouch-analen Adenokarzinoms zu beachten, welches aus residueller Rektumschleimhaut oder Mukosa des oberen Analkanals hervorgeht, die bei der Pouch-Operation hinterlassen wurde. Dies gilt nicht nur für jene Operationsvarianten, welche auf eine Proktomukosektomie verzichten. Auch nach einer Proktomukosektomie bis zur Linea dentata verbleiben öfters quadratmillimeterkleine Reste von Rektumtypschleimhaut des oberen Analkanals und damit hinterbleibt ein kleines Krebsrisiko. Die bisher beobachteten Fälle eines Pouch-analen Adenokarzinoms sind bei Colitis-ulcerosa-Patienten aufgetreten, die schon vorher im Kolon ein Karzinom oder Dysplasien hatten. Entsprechend gelten die gleichen Risikoindikatoren wie beim Colitis-ulcerosa-assoziierten Darmkrebs.

Ein anderes Risiko stellt ein Adenokarzinom im Pouch-Ileum dar. Die wenigen bisher bekannten Einzelfälle lassen noch unsicher definieren, welche Risikokonstellation hierfür vorliegt. Dazu dürften eine vorbestehende retrograde Anschlussileitis gehören und die seltenen Fälle einer Pouchitis mit atrophischer Mukosa. Die bioptische Diagnostik von präkanzerösen Dysplasien im Pouch-Ileum ist schwieriger als in Kolon und Analkanal.

Fölsch: Die Risikokonstellationen zur Entwicklung eines Pouch-Karzinoms sind aufgrund der kleinen bisher beobachteten Fallzahlen noch sehr unklar. Ein langjähriger «pouchitischer» Krankheitsverlauf gehört hier sicherlich dazu. Weiterhin kann sich ein Karzinom in diesem Bereich auch aus Res- 
ten analer Schleimhaut entwickeln. Unsere Patienten werden in ein Überwachungsprogramm eingeschlossen, das auch regelmäßige Pouchoskopien beinhaltet. In jedem Fall darf man nicht davon ausgehen, dass mit der operativen «Beseitigung» der Grunderkrankung (d.h. Proktokolektomie und ileoanale Pouch-Anlage) kein Betreuungsbedarf mehr gegeben ist.

Layer: Patienten mit schwerer Zottenatrophie sind besonders gefährdet, ein Pouch-Karzinom zu entwickeln. Bei ihnen sollte eine Dysplasiediagnostik mit Stufenbiopsien mindestens jährlich erfolgen. Bei gleichzeitigem Vorliegen einer primär sklerosierenden Cholangitis bzw. bei vorangegangener Backwash-Ileitis könnte das Risiko zusätzlich erhöht sein; dies ist aber noch nicht eindeutig geklärt. Hochtitrige perinukleäre antineutrophile zytoplasmatische Antikörper vor Proktokolektomie sollen einer aktuellen Studie zufolge einen besonderen Risikofaktor für die Entwicklung einer chronischen Pouchitis und damit mittelbar eines Pouch-Karzinoms darstellen. Dieser Parameter könnte zukünftig klinische Bedeutung erlangen.

Bruch/Gehl/Eckmann: In einer Subgruppe von Patienten nach totaler Kolektomie entwickelt sich bei chronischer Pouchitis eine schwere und persistierende villöse Atrophie einer kolonartigen Mukosa (Typ C). Dysplasie und Aneuploidie auf chromosomaler Ebene reflektieren verschiedene Wege einer möglichen Karzinomentstehung. Pathophysiologisch liegen der erhöhten Dysplasiefrequenz vermutlich eine chronische Entzündung und eine gesteigerte Proliferation zugrunde. PouchitisKarzinome sind selten, aber in den letzten 5 Jahren beschrieben. Daher sind engmaschige endoskopisch-bioptische Kontrollen bei Patienten mit persistierender chronischer Pouchitis dringend erforderlich. Besteht eine chronische Pouchitis über 10 Jahre und treten bestätigte gering- oder neue hochgradige Dysplasien auf, ist das Risiko einer malignen Entartung so groß, dass die Indikation zur Entfernung des Pouches gegeben ist.

\section{Frage 9: Welche Ausnahmeindikationen sehen Sie für eine subtotale Kolektomie und eine ileorektale Anastomose in der chirurgischen Therapie der Colitis ulcerosa?}

Fölsch: Die ileorektale Anastomose ist die einzige Alternative zur vollständigen Proktokolektomie und sollte unseres Erachtens nur bei Patienten eingesetzt werden, deren Grunderkrankung nicht einer typischen Colitis ulcerosa entspricht. Wir überblicken wenige Patienten mit einer Colitis indeterminata, die vom endoskopischen Beschwerdebild weitgehend einer Colitis ulcerosa entsprachen, deren Rektum jedoch ohne vorherigen Einsatz rektaler topischer Therapie entzündungsarm oder entzündungsfrei war. Bei diesen Patienten haben wir die Empfehlung zu einer Ileorektalanastomose erfolgreich mitge- tragen. Bei einer typischen Colitis ulcerosa (auch wenn sie nur linksseitig verläuft) sehen wir keine Indikation für ein subtotales Kolektomieverfahren oder für eine ileorektale Anastomose.

Layer: Eine Kolektomie mit Ileorektostomie kann zumindest als vorläufige Maßnahme bei Männern mit noch nicht abgeschlossener Familienplanung durchgeführt werden, da bei diesem Operationsverfahren das Risiko von Potenzstörungen deutlich geringer ist als bei der Proktokolektomie. Eine komplette Impotenz ist zwar auch nach Proktokolektomie mit etwa $2 \%$ selten, jedoch werden Potenz- bzw. Blasenentleerungsstörungen in 5-30\% der Fälle berichtet.

Bruch/Gehl/Eckmann: Aus onkologischer Sicht ist bei der Colitis ulcerosa immer eine totale Kolektomie erforderlich. Unstreitig ist, dass im Bereich einer verbleibenden Rektummukosa nach ileorektalen Anastomosen vermehrt Stumpfkarzinome nachgewiesen wurden. Grundsätzlich ist die subtotale Kolektomie also keine Therapie der Wahl bei der Colitis ulcerosa. Nur in wenigen Ausnahmefällen kann sie erwogen werden: 1. Bei jungen Menschen mit Kinderwunsch, die nur gering- oder mittelgradige Dysplasien oder Ernährungsstörungen aufweisen, besteht bei der subtotalen Kolektomie mit ileorektaler Anastomose der Vorteil, dass die anatomischen Strukturen, die für die Sexualfunktion wichtig sind, sicher geschont werden. Bei diesen Patienten ist allerdings eine sehr genaue endoskopische Kontrolle in regelmäßigen Abständen nötig. 2. Bei Patienten über 70 Jahren und fortgeschrittenem Tumorstadium sowie eingeschränktem Allgemeinzustand kann die subtotale Kolektomie dann von Vorteil sein, wenn das Überleben limitiert ist. Hier können lokale Probleme (Pouchitis) oder hohe Flüssigkeitsverluste nach Anlage einer Ileostomie die Lebensqualität während der ohnehin verkürzten Lebenszeit beeinträchtigen.

\section{Teilnehmer}

Prof. Dr. A. von Herbay
Pathologisches Institut
Universitätsklinikum Heidelberg
Im Neuenheimer Feld 220
D-69120 Heidelberg (Deutschland)
Tel./Fax 06221562675
E-mail pathologe@vonherbay.de
Prof. Dr. Brambs
Radiologische Klinik
Universitätsklinikum Ulm
Steinhövelstraße
D-89075 Ulm (Deutschland)
Tel. +49 731 500 274-00, Fax -09
E-mail hans-juergen.brambs@medizin.uni-ulm.de
Prof. Dr. U.R. Fölsch
Klinik für Allgemeine Innere Medizin
I. Medizinische Universitätsklinik
Schittenhelmstraße 12
D-24105 Kiel (Deutschland)
Tel. +49 $4315971-271$, Fax -302
E-mail urfoelsch@med.uni-kiel.de

Prof. Dr. P. Layer

Abteilung für Innere Medizin

Israelitisches Krankenhaus

Orchideenstieg 14

D-22297 Hamburg (Deutschland)

Tel. +49 405112 52-11, Fax -27

E-mail layer@ik-h.de

Prof. Dr. H.-P. Bruch

Dr. H.-B. Gehl, Dr. C. Eckmann

Klinik für Chirurgie

Universitätsklinikum Lübeck

Ratzeburger Allee 160

D-23538 Lübeck (Deutschland)

Tel. +49 45150 020-01, Fax -69

E-mail bruch@medinf.mu-luebeck.de 